La implementación del Plan FinEs: un análisis desde el trabajo docente

Maia Gruszka, Ariadna Abritta

http://perio.unlp.edu.ar/ojs/index.php/question/article/view/4717

Cita sugerida: Gruszka, M., y Abritta, A. (2018). La implementación del Plan FinEs: un análisis desde el trabajo

docente. Question, 1(59), e072. doi:https://doi.org/10.24215/16696581e072

\title{
La implementación del Plan FinEs: un análisis desde el trabajo docente
}

The FinEs Plan implementation: an analysis since the teaching work conditions

Maia Gruszka mai.grusz@gmail.com

http://orcid.org/0000-0003-1815-9784

Facultad de Ciencias Sociales; Universidad de Buenos Aires (Argentina)

Ariadna Abritta ariadna.abritta@gmail.com

http://orcid.org/0000-0002-8557-9669

Escuela de Política y Gobierno; Universidad Nacional de San Martín/

Universidad de Buenos Aires (Argentina)

\section{Resumen}

El Plan de Finalización de Estudios (FinEs) fue diseñado en 2008 por el Ministerio de Educación de la Nación como un instrumento de política pública tendiente a mejorar las oportunidades de culminación de los estudios obligatorios para la población joven y adulta que no pudo realizarlo en los tiempos que el sistema escolar establece como normales. Dicho programa socioeducativo adquirió características particulares según la jurisdicción en la que se implementó como así también según el recorte temporal que se analice. 
La proliferación de programas que se situaron en los bordes del sistema educativo generó la instalación de nuevos debates en torno a la inclusión y la calidad educativa, y también sobre las condiciones de trabajo docente asociadas.

El presente artículo busca analizar algunas de las tensiones que se registraron en los posicionamientos que orientaron la implementación del Plan Fines y profundizar en torno a la dimensión del trabajo docente en dicho programa. Siendo los planes y programas educativos uno de los ejes centrales de la política educativa de la Argentina en el período 2003-2015, con este artículo esperamos aportar elementos que permitan complejizar los análisis al prestar atención a las dinámicas concretas que adquirió la implementación de dichas iniciativas.

Palabras clave: políticas educativas; programas socioeducativos; implementación; trabajo docente.

\section{Abstract}

The plan for completion of studies (FinEs, for his acronym in spanish) was designed in 2008 by the National Ministry of Education as an instrument of public policy aimed at improving the opportunities for completion of compulsory studies by the young and adult population who couldn't do it at the time that the scholar system establish as normal. This socioeducational programme acquired particular characteristics according to the jurisdiction it was implemented as well as the temporal cut that is analyzed.

The proliferation of educational programs that were located at the edges of the educational system generated the installation of new debates around inclusion and educational quality, and the associated working conditions of teachers.

This article seeks to analyze some of the tensions that were recorded in the positions that guided the implementation of Fines Plan and focus into the dimension of teaching work in that program. Since the educational plans and programs were central axes of the education policy in the Argentina of 2003-2015, this article hopes to provide elements that will make the analysis more complex by paying attention to the specific dynamics acquired by the implementation of those initiatives.

Keywords: educational policies; socioeducational programmes; implementation; teaching work.

El Plan de Finalización de Estudios (FinEs) fue diseñado en 2008 por el Ministerio de Educación de la Nación (MEN) como un instrumento de política pública tendiente a mejorar las 
posibilidades de culminación de los estudios obligatorios por parte de la población joven y adulta que no lo pudo realizar en los tiempos que el sistema escolar estipula como "normales". La proliferación de políticas y programas educativos que se situaron en los bordes del sistema generaron la instalación de nuevos debates en torno a la inclusión y la calidad educativa, sobre las condiciones de trabajo docente asociadas a los "programas a término", y en lo relativo a los posicionamientos discursivos que orientaron el diseño y la implementación de estas políticas. Todas estas temáticas atravesaron tensiones diversas y particulares.

En el caso del Plan FinEs el mismo fue asimismo adquiriendo características específicas según la jurisdicción que se tome en consideración como así también a partir el recorte temporal que se analice. Así podemos identificar dinámicas diferenciales de implementación entre la Ciudad Autónoma de Buenos Aires (CABA) y la provincia homónima (PBA).

A pesar de las diferencias en tamaño, población, cantidad de personas con estudios obligatorios inconclusos y ofertas educativas destinadas a jóvenes y adultos que se registran entre ambas jurisdicciones, nos parece relevante el análisis comparativo de las dinámicas de implementación del FinEs en CABA y PBA por, al menos, tres motivos:

1. Por la visibilidad y peso político que tienen estas dos jurisdicciones en el nivel nacional. De hecho, las realidades y dinámicas que asumen las políticas en estas dos jurisdicciones construyen usualmente representaciones y sentidos que "adquieren" alcance nacional.

2. Por las diferencias en el signo político partidario que se expresan entre ambas jurisdicciones en el período 2008-2015, y que puede ser un factor interviniente a la hora de analizar los motivos que fundamentan las dinámicas diferenciales que adquirió la implementación del Plan FinEs.

3. Por las prácticas de movilidad docente y estudiantil que históricamente se registran entre ambas jurisdicciones.

Así, el presente artículo busca abordar y complejizar los debates en torno a las condiciones del trabajo docente en el Plan FinEs desde múltiples dimensiones: por un lado, desde las diferencias que se plasmaron en relación a los/as destinatarios/as y los emplazamientos de las comisiones del programa, que permiten contextualizar su alcance y las características territoriales que guiaron su implementación. Por el otro, las diferentes regulaciones que se establecieron sobre el procedimiento de selección docente y las condiciones de trabajo asociadas. Finalmente, las diversas formaciones docentes y estrategias de capacitación que se plantearon para estos profesores/as desde la perspectiva de la educación popular. 
Para dar cuenta de todo esto, en términos metodológicos, se realizó un análisis de la normativa de orden nacional y jurisdiccional que regulaba el funcionamiento de este programa educativo y se recurrieron a datos secundarios disponibles en distintos trabajos académicos y en fuentes periodísticas a los efectos de dimensionar el alcance territorial y tasas de matriculación que el programa presentaba a 2015. Asimismo se realizaron análisis de entrevistas en profundidad a docentes, inspectores, estudiantes y funcionarios públicos comprometidos con la implementación local del FinEs.

El objetivo general que se busca es realizar una descripción densa de los procesos de implementación local de este programa educativo intentando dar cuenta de su heterogeneidad interna y de las valoraciones diferenciales de los actores implicados hasta 2015. En síntesis, este trabajo se propone analizar la dimensión del trabajo docente en el Plan FinEs con la intencionalidad de complejizar y aportar nuevas consideraciones a los debates en torno los planes y programas educativos que fueron ejes centrales de la política educativa de la Argentina en el período 2003-2015.

\section{Aportes teóricos de los estudios sobre políticas públicas}

Partimos de comprender a las políticas públicas como una de las formas en que el Estado se materializa, es decir, cobra forma, contenido, y proximidad con los ciudadanos (O'Donnell y Oszlak, 1981). Estos autores conceptualizan a la política pública (o estatal, según sus propios términos) como una tentativa de intervención sobre una "cuestión", es decir un asunto, entendido como necesidades y demandas socialmente problematizadas, sobre el cual se despliegan conflictos, coaliciones, poder, evidencias y argumentaciones.

Así O’Donnell y Oszlak plantean que las políticas estatales se insertan en una "estructura de arenas" que debemos conocer mejor para entender por qué se plantean y resuelven cuestiones de unas $u$ otras maneras. Concomitantemente resaltan la importancia del análisis de los distintos actores intervinientes en torno a la problematización de una cuestión social, los cuales tienen distintas cuotas de poder y capacidad de incidencia sobre el Estado.

Asimismo los autores resaltan que la toma de posición del Estado (a través de las políticas públicas) no siempre resulta unívoca, homogénea o permanente. Las predisposiciones o decisiones de las diversas instancias intervinientes resultan a menudo inconsistentes o conflictivas entre sí. En síntesis, acordamos con la visión del carácter negociado o 
abiertamente conflictivo que frecuentemente asumen la toma de posición del Estado y las políticas públicas que plantea frente a una cuestión social.

Por su parte, Tamayo Sáez (1997) también recupera esta perspectiva temporal y secuencial de las políticas públicas y plantea el carácter cíclico de las mismas. En este sentido, este autor argumenta que los resultados de la política retroalimentan los procesos de diseño e implementación de estas u otras políticas. Esta retroalimentación no requiere necesariamente de la evaluación explícita o formal de los resultados, sino que se pueden generar cambios en el diseño o en la implementación misma a partir de las observaciones iniciales de los efectos de la puesta en funcionamiento de una política.

Finalmente Aguilar Villanueva (2007) plantea que la política pública es el resultado de las decisiones y también de las interacciones que comportan estas decisiones. Dentro de estas interacciones y posiciones heterogéneas dentro del Estado mismo podemos identificar tanto a las que se dan entre las diversas agencias estatales de un mismo nivel de gobierno como así también a aquellas que se plantean entre diferentes niveles de gobierno.

Nos parece relevante retomar los aportes de estos autores para desde allí poder hacer énfasis tanto en las disputas y conflictos entre diferentes actores como así también en las orientaciones, intereses y necesidades que impregnan tanto el diseño como la implementación de las políticas públicas.

\section{Breve recorrido por la historia de la Educación de Jóvenes y Adultos en la Argentina}

La Educación de Jóvenes y Adultos (EDJA) se ha caracterizado a lo largo de su historia por la búsqueda de estrategias y emplazamientos que permitan vincular la vida productiva y reproductiva de sus sujetos destinatarios con los dispositivos formativos que se proponen. En este sentido, la proximidad física de los espacios destinados a la formación con los lugares de trabajo y/o residencia de los jóvenes y adultos ha sido una nota distintiva de la EDJA a lo largo de su historia.

En 1968 se crea en el ámbito nacional la Dirección Nacional de Educación de Adultos (DINEA) que tuvo a su cargo las acciones educativas de la modalidad hasta su cierre en 1992. La creación de esta agencia estatal representa una jerarquización de las acciones educativas destinadas a los jóvenes y adultos. Podemos identificar esta orientación hacia la inserción comunitaria de la EDJA que mencionamos anteriormente en las experiencias de la DINEA en el período 73-74 (Rodríguez, 1997) y, más específicamente, en la creación de los Centros de 
Educación de Nivel Secundario (CENS) mediante la celebración de convenios con organizaciones laborales, sindicales, y barriales (Cabrera, 2006).

Existe una amplia producción académica en torno a los efectos nocivos de las reformas estructurales aplicadas en el ámbito educativo en toda América Latina durante los años de hegemonía del modelo neoliberal (Gentili, 1998). En el caso argentino estas reformas se caracterizaron, a grandes rasgos, por una reducción de las responsabilidades del Estado nacional en materia educativa, por procesos de descentralización descoordinada de las unidades escolares en las provincias, el desfinanciamiento de los sistemas educativos provinciales, el aumento de las tasas de no terminalidad primaria, así como por la precarización de las condiciones laborales de los docentes, y el deterioro de la infraestructura escolar, entre otros elementos (Feldfeber, 1997). Estas orientaciones adquirieron institucionalidad y formalización a través de la Ley Federal de Educación Nro. 24195 de 1993, que entre otras modificaciones, establece como "regímenes especiales" a la Educación Especial, la Educación de Adultos y la Educación Artística.

En 2006 se sancionará la Ley Nacional de Educación (LEN) Nro. 26606 la cual, entre otros aspectos, establecerá la extensión de la educación obligatoria, incluyendo al nivel medio, y rejerarquizará a la educación de jóvenes y adultos que dejará de ser considerada un "régimen especial" para volver a ser una modalidad educativa. Es en dicho contexto de ampliación de la obligatoriedad educativa y de re-jerarquización de la educación de jóvenes y adultos que el Plan FinEs se diseñará.

\section{Características generales del Plan FinEs}

El Plan de Finalización de Estudios (FinEs) surge como iniciativa del Ministerio de Educación de la Nación en 2008 con un plazo de implementación acotado, en su carácter de programa temporal "a término" (LEN: art. 138). Según su resolución de origen (Res. CFE. 66/08) la implementación del programa comprendería el período 2008-2011, y se buscaba que tuviera alcance nacional y funcionará en todas las jurisdicciones.

En su origen el Plan FinEs tenía como objetivo ampliar las posibilidades de finalización de estudios a aquellas personas que habían culminado la cursada de los estudios primarios o secundarios pero no habían alcanzado la certificación correspondiente por adeudar la aprobación de un número limitado de materias. La modalidad consistía entonces en la preparación y acompañamiento, a través de tutorías, para la rendición de exámenes finales de 
determinadas materias. Es a esta modalidad del plan que luego se conocería como "Fines 1" o "Fines puro".

Hacia 2009 el Ministerio de Desarrollo Social de la Nación (MDS) impulsa la creación del programa "Ingreso Social con Trabajo-Argentina Trabaja" (PRIST-AT), que incluía entre sus componentes una línea interna vinculada a la educación. Así, una de las primeras actividades planteadas por el equipo educativo del PRIST-AT consistió en la realización de talleres y de una encuesta para conocer la situación educativa de los y las cooperativistas inscriptas en el Programa. Los resultados de las más de 35.000 encuestas realizadas en los 32 distritos del conurbano bonaerense que se seleccionaron para la primera etapa de implementación del programa permitieron identificar como principal problemática la terminalidad educativa, especialmente de nivel secundario.

Es así que en el año 2010 se diseña e implementa mediante la Resolución Nro. 3520/10 de la Dirección General de Cultura y Educación (DGCyE) de la provincia de Buenos Aires (PBA) el Plan FinEs 2 (o "FinEs trayecto" según su denominación coloquial), una variante del Plan FinEs original, destinado específicamente a los y las cooperativistas del PRIST-AT que no hayan finalizado sus estudios secundarios.

Esta nueva modalidad del Plan estipulaba no solamente la preparación para rendir materias adeudadas por aquellos que habían culminado el cursado de sus estudios secundarios en calidad de alumnos regulares, sino también una propuesta específica para aquellos que no hubieran podido comenzar o finalizar la cursada de sus estudios secundarios. El Plan FinEs 2 promovió la constitución de centros de terminalidad educativa en los establecimientos que las cooperativas y/o las organizaciones sociales de las que formaban parte contaban. En términos pedagógico-organizativos, el Plan FinEs 2 contempla el cursado total del nivel medio de adultos por parte de los/as cooperativistas, con un sistema semi-presencial de dos días de cursada semanal, y con una duración total de seis cuatrimestres o tres años, pudiendo dar inicio el ciclo lectivo en ambos cuatrimestres.

Vale la pena recordar que en la PBA ya existía previamente una propuesta similar a la planteada por el FinEs 1, denominada Centros de Orientación y Apoyo (COA) (1). Dichos centros promueven el acompañamiento, mediante un sistema de tutorías, para la preparación de exámenes, la rendición de materias adeudadas, y el acceso a la titulación de nivel secundario. Por eso a partir de la creación del FinEs 1 estas propuestas pasarán a conocerse como FinEs-COA. A su vez, desde 2007 se implementaba en la provincia un Bachillerato a distancia para Jóvenes y Adultos (Res. DGCyE Nro. 737/07), es decir, una propuesta con formato de asistencia semi presencial, a partir de una organización curricular modular, las 
cuales constituyen propuestas organizativas predominantes en las tendencias actuales para la formación/educación de jóvenes y adultos (Misirlis, 2009).

En abril de 2012, mediante Resolución DGCyE 444/12, se resuelve en el ámbito de la provincia de Buenos Aires la ampliación de los destinatarios del Plan FinEs 2 al conjunto de los jóvenes y adultos -mayores de 18 años- que no hayan podido comenzar y/o concluir sus estudios secundarios. Asimismo, en dicho año, se sanciona la disposición SE-DGCyE 99/12 por la cual se reconocen los trayectos educativos previos de los estudiantes con independencia de la culminación del nivel. Se trata en otros términos de un mecanismo de equivalencias de materias y años de estudios antecedentes.

Posteriormente se generarán nuevas articulaciones con otros programas sociales, como el Programa PROG.R.ES.AR (2) y el "Ellas Hacen" (3), que contemplan como una de las contraprestaciones asociadas a los subsidios monetarios percibidos la finalización de los estudios obligatorios. De esta forma el Plan Fines se consolidará como la línea educativa principal de diversos programas sociales. A esta propuesta de articulación y coordinación de distintas líneas y programas sociales el MDS la denominará "Políticas Sociales Integrales" (Kirchner, 2007).

A pesar de que en el seno de la Provincia existían diversas propuestas educativas para la población de jóvenes y adultos con estudios inconclusos, el Censo Nacional de 2010 indicó que existían aproximadamente 4.400 .000 personas con estudios secundarios incompletos en dicha jurisdicción. De este universo, las propuestas de EDJA de la provincia -entre los que se encuentran los históricos CENS y los Bachilleratos de Adultos (4)- solo alcanzaban a unos 230.000 jóvenes (5). Para 2011, es decir en su segundo año de implementación, estudiaban en el Plan FinEs 2 o Fines trayecto más de 15.000 estudiantes, que llegarán en 2015 a ser más de 100.000, distribuidos en más de 6.000 comisiones constituidas en 126 de los 135 distritos que componen la PBA. Estos 100.000 jóvenes y adultos se incorporarán a los estudios secundarios aumentando significativamente el alcance de las propuestas de la modalidad, no registrándose variaciones significativas -ni por lo tanto movilidad- en la matrícula de los servicios educativos de nivel secundario para jóvenes y adultos entre 2008 y 2014 (6).

Por su parte, en el ámbito de la Ciudad Autónoma de Buenos Aires (CABA), en 2008 el Gobierno local firma la Resolución 8644-MEGC/08 y al año siguiente la Resolución 3789MEGC/09, en las que se dispone la implementación del Plan FinEs 1 o FinEs puro como primera etapa. Años más tarde, a través de la Resolución 5858-MEGC/11, también adscribirá a la implementación del FinEs 2 o FinEs Trayecto. 
El impacto del Plan FinEs en CABA ha sido notoriamente distinto respecto al registrado en la provincia de Buenos Aires. En primer lugar, como ya se mencionó, el plan FinEs 2 surge en la provincia como un resultante de la articulación entre el MDS y la DGCyE en función de promover la terminalidad educativa de los cooperativistas del PRIST-AT (7), programa social que no llegó a implementarse en ninguna de sus etapas en CABA. En segundo lugar la implementación del Plan Fines en CABA no ha contado hasta el momento con el impulso del gobierno local, ya sea por cuestiones político-partidarias (8), o por contar con menor población con estudios obligatorios inconclusos (9) a la par de una mayor cobertura de servicios educativos propios para la modalidad de jóvenes y adultos, y programas específicos como "Adultos 2000" (10).

Analizando el Plan FinEs como ejemplo de política pública educativa de nivel nacional, y comparando su implementación en la PBA y en la Ciudad Autónoma de Buenos Aires durante el período 2008-2015, resulta visible que existen múltiples diferencias entre ambas jurisdicciones. Es a las dinámicas diferenciales de implementación del Plan FinEs entre ambas jurisdicciones y a las condiciones particulares de selección, trabajo y capacitación de sus trabajadores docentes que dedicaremos las próximas líneas.

\section{Análisis de las condiciones diferenciales de trabajo docente en el Plan FinEs en CABA y PBA}

Como se sostuvo en la introducción de este trabajo, el Plan FinEs ha captado la atención y ha protagonizado diversos debates tanto en el campo educativo como en los medios masivos de comunicación. Las críticas hacia este programa socioeducativo han sido hasta ahora encaradas principalmente desde el aspecto salarial y de estabilidad laboral de los docentes, por un lado, y sobre la calidad de la propuesta pedagógica, por el otro. Asimismo dichas críticas han sido enunciadas tanto por parte de algunos sectores del sindicalismo docente, como por miembros de experiencias autogestivas de EDJA, entre otros.

En cuanto al rol de los sindicatos docentes respecto a los trabajadores del Plan FinEs, su comportamiento ha sido, como mínimo, oscilante y ambivalente. Rescatando los logros del Plan FinEs e incentivando su implementación, no han tenido respecto a los trabajadores docentes del FinEs una propuesta de protección sindical clara.

Por otro lado, cabe mencionar las controversias que la implementación del Plan FinEs ha suscitado entre los trabajadores docentes de los CENS y de los Bachilleratos de Adultos del 
sistema formal de educación de jóvenes y adultos. Los mismos han generalmente criticado al FinEs por su organización semi-presencial y por sus formatos de evaluación, a los cuales se suele asociar a una baja calidad educativa. Respecto a este último punto, vale la pena recuperar los múltiples trabajos sobre alternativas pedagógicas y educación popular que promueven otras modalidades de evaluación, mediante producciones creativas, que impulsen procesos de pensamiento crítico y de problematización del mundo en el cual nos encontramos inscriptos (Freire, 2002). La educación popular como propuesta pedagógica promueve otra trama de sentidos y otros fines para la educación que requieren inevitablemente problematizar visiones tecnocráticas de la evaluación y perspectivas enciclopedistas en torno a la calidad educativa.

Lo que es innegable es que el FinEs ha resultado relevante y ha llamado la atención tanto para quienes lo reivindican por sus posibilidades de garantizar derechos históricamente vulnerados (aquellos que lo identifican como una alternativa pedagógica), como por quienes han analizado sus limitaciones en relación a las condiciones de trabajo docente o han cuestionado los parámetros de "calidad" de la propuesta. En todos los casos nos parece que una de las potencias del Plan FinEs es su capacidad problematizadora que ha abierto diversos debates. A continuación proponemos algunas dimensiones de análisis que nos permiten conocer las formas diferenciales de implementación que adquirió esta política educativa en CABA y en la PBA en el período 2008-2015 y las distintas situaciones laborales de sus trabajadores.

\section{a. Los/as destinatarios/as y los emplazamientos diferenciales entre CABA y PBA}

Como se ha expresado anteriormente, en la provincia de Buenos Aires la implementación inicial del Plan FinEs 2 se desarrolló en articulación con el PRIST-AT. De esta forma el comienzo del Fines Trayecto estuvo planificado para los cooperativistas del "Argentina Trabaja" y el desarrollo del mismo se preveía en las mismas sedes de funcionamiento de las cooperativas. Luego, según las entrevistas realizadas, se acercaron a las sedes del FinEs familiares y vecinos de los titulares del PRIST-AT consultando sobre la posibilidad de participar de esta propuesta de terminalidad educativa. Así comienza a modificarse el diseño de la política y abrirse la posibilidad de inscripción a otros miembros de la comunidad (11).

Esta articulación con el programa PRIST-AT va a marcar la implementación y el desarrollo del Plan FinEs en la provincia de Buenos Aires, en el que el MDS participará activamente; dotando de características particulares al Plan, principalmente en relación a los emplazamientos de las comisiones que se darán en su mayoría en sedes comunales, clubes de barrio, espacios cedidos por organizaciones sociales, o por la propia comunidad. 
La situación en CABA fue diferente. En esta jurisdicción el programa PRIST-AT no ha tenido desarrollo. La implementación del Plan FinEs se realizó a partir de los convenios firmados entre organizaciones sociales y sindicales con el Ministerio de Educación de la Nación, por lo que la apertura de comisiones y sedes se realizaba por indicación de este, y asumiendo el Gobierno de la Ciudad la supervisión y posterior administración de recursos, emisión de certificados, etcétera.

A su vez, las disímiles características estructurales y territoriales entre la PBA y la CABA son ineludibles y mientras colaboran a explicar las diferencias en el impacto del Plan FinEs complejizan el análisis comparativo aquí propuesto (12). En el ámbito de la provincia de Buenos Aires existían, hasta 2015, 125 CENS en 56 distritos, es decir, más de la mitad de los distritos de la provincia no contaban con servicios educativos propios de la modalidad de jóvenes y adultos. Por su parte, en CABA existían más de noventa CENS distribuidos en todas las comunas en la que se divide administrativamente la ciudad. Estos números ilustran y explican algunas de las diferencias en la cantidad de comisiones abiertas del plan FinEs en cada jurisdicción y en el impacto diferencial del programa.

\section{b. Selección docente e ingreso al Plan FinEs}

En la PBA el mecanismo de ingreso al Plan FinEs como trabajador docente se realiza mediante acto público, es decir, mediante el mecanismo que organiza y regula el ingreso a la docencia en todo el sistema educativo. La diferencia específica del FinEs se encuentra en la forma particular de inscripción que establece y en las características de la evaluación de antecedentes y propuestas para la construcción de los listados y el puntaje docente.

Para trabajar en el Plan FinEs 2 se estipula un plazo específico de inscripción y se debe presentar además de un curriculum vitae con la documentación respaldatoria y un proyecto pedagógico por cada una de las asignatura que se aspira a dictar(13). Asimismo para la elaboración de los proyectos pedagógicos se incentiva a los docentes a plantear propuestas de trabajo inscriptas en la perspectiva de la educación popular (Resolución DGCyE 3520/10). La presentación de un proyecto pedagógico resulta un aspecto novedoso en relación al acceso tradicional a cargos dentro del sistema de educación público. La evaluación de estos antecedentes y propuesta, la realiza el inspector de adultos y la mesa de trabajo distrital en la que tienen representación los trabajadores de MDS y distintos referentes barriales involucrados con la implementación del Plan. Según las entrevistas realizadas en pocos distritos se desarrolló este proceso de la forma establecida y en la mayoría de los casos la evaluación y 
realización del orden de mérito fue asumida principal -y casi únicamente- por el cuerpo de inspectores de la modalidad.

En CABA los docentes que se desempeñan en el Plan FinEs también fueron designados por la normativa vigente y dependen, así como todo el programa, de la Dirección de Adultos del Ministerio de Educación porteño. La diferencia sustancial con la PBA es que el acceso a estos cargos se realiza a través de la inscripción en la Junta de Clasificación Docente de la modalidad de Adultos, quien asigna puntaje a los antecedentes y confecciona un orden de mérito único para toda la modalidad, ya sea para el trabajo en CENS o dentro del Plan FinEs. Asimismo, a diferencia de la provincia, en CABA no hay una instancia de presentación de un proyecto pedagógico por parte de los docentes en vistas a considerar la especificidad pedagógica y territorial que orienta al Plan.

Así, el mecanismo de selección docente propuesto por el Gobierno de CABA (modelo de selección tradicional basado en antecedentes y puntaje) pareciera dificultar tomar en cuenta aspectos como el compromiso docente y la elaboración de una propuesta pedagógica acorde a las características socioculturales y territoriales que caracterizan al FinEs.

\section{c. Condiciones salariales, licencias estatutarias y estabilidad laboral}

En relación a las condiciones de trabajo docente tanto en CABA como en la PBA los fondos requeridos para la cobertura de los salarios docentes dependían del presupuesto nacional asignado al Ministerio de Educación de la Nación, quien luego transfería los recursos a las jurisdicciones según el tamaño de la planta docente del Plan FinEs en cada una de ellas. Este elemento abre algunos interrogantes en relación a las diferencias salariales que se registraron entre las jurisdicciones.

En la PBA los docentes del Plan FinEs recibieron desde el comienzo haberes similares a los de los docentes de CENS; el mismo monto, la misma forma de liquidación y con las mismas condiciones salariales (antigüedad, aguinaldo, vacaciones, garantía mínima, etcétera). Por su parte, en CABA el salario docente de los trabajadores del plan FinEs estuvo congelado entre 2013 y 2015, y las liquidaciones no respetaban las mismas condiciones ni ítems que los trabajadores de la modalidad de jóvenes y adultos; no se contemplaba antigüedad, ni aguinaldo ni la garantía mínima.

Otro elemento central en relación a las condiciones de trabajo docente que han sido eje de las críticas está vinculado con la imposibilidad de gozar de los derechos de licencias estatutarias y la estabilidad laboral, ya que los docentes eran designados por cuatrimestre. En relación a este 
último aspecto es necesario precisar que, como ya se mencionó, el Plan FinEs es un programa a término, lo cual dificulta las formas de alcanzar esta estabilidad laboral demandada.

Transcurridos varios años de implementación del Plan, puede notarse un recambio de aspirantes a tomar horas dentro del FinEs, pero también un grupo de profesores relativamente estable que vienen trabajando en su implementación con regularidad. Así, en la esfera del trabajo docente del Plan FinEs, encontramos docentes que hacen su primera experiencia de trabajo profesional, docentes que lo contemplan como una segunda opción laboral (dadas las diferencias salariales y de condiciones laborales con otros espacios educativos), y también un cuerpo estable de docentes que se desempeñan en el Plan FinEs por motivaciones que no pueden atribuirse solamente a la necesidad salarial sino que consideramos está íntimamente imbricada con la orientación político pedagógica de la que se intenta dotar su práctica.

\section{d. Propuestas de formación docente continua en el Plan FinEs}

Finalmente, la falta de formación docente específica es una problemática histórica de la modalidad de educación de jóvenes y adultos, ya que salvo cursos o postítulos electivos, no hay propuestas específicas y formales de formación docente para la escuela primaria o media de adultos.

El Plan FinEs manifiesta en sus múltiples documentos -nacionales y de la PBA- una orientación pedagógica hacia la educación popular y asimismo se insta a los docentes a presentar propuestas que se inscriban en esta perspectiva educativa; sin embargo, las capacitaciones e instancias de formación docente que se ofrecen en el marco del Plan resultan aún insuficientes. Sin embargo, también aquí encontramos divergencias entre las propuestas desarrolladas por el gobierno de la PBA respecto al de CABA.

Durante 2014 y 2015, la Dirección de Educación de Adultos de la PBA promovió la realización de una serie de cursos destinados a docentes de la modalidad de jóvenes y adultos y del Plan FinEs con la perspectiva de promover la formación específica para el trabajo con estos sujetos pedagógicos, encuadrados en la perspectiva de educación popular. Los mismos se desarrollaban principalmente los días sábados con el objetivo de facilitar la participación de los docentes y otorgaban puntaje docente. Respecto a CABA no se han podido registrar iniciativas similares (independientemente del enfoque pedagógico propuesto). 


\section{Palabras finales}

Las opciones de inclusión social y educativa que se propusieron en el período 2003-2015 plantearon nuevas tensiones y desafíos en torno al trabajo docente dentro de programas socioeducativos que adquieren particular relevancia en el contexto actual de desestructuración y retroceso en materia de derechos y condiciones laborales. El Plan FinEs es un ejemplo de ello, ya que evidencia los debates que se generaron entre diversas cuestiones tales como la "terminalidad" de los programas a término contemplados en la LEN, la conformación de listados específicos para la selección docente, la inclusión de proyectos pedagógicos para la conformación del puntaje, las condiciones salariales contempladas en los programas educativos, entre otros.

Hasta aquí hemos desarrollado una breve comparación en relación a los aspectos centrales del trabajo docente del Plan FinEs en la PBA y en CABA. De esta comparación nos resulta interesante compartir algunas reflexiones:

-Los sentidos político pedagógicos, y en particular su vinculación con la perspectiva de la educación popular, que le otorgaron cada una de las jurisdicciones marca diferencias sustanciales en las dinámicas de implementación de un mismo programa. Algunos de los aspectos en lo que esto puede visibilizarse es quiénes son y cómo se comprende a los destinatarios de los programas educativos, cuáles son los espacios físicos o territoriales en los cuales se emplazan dichos programas, y cuál es el lugar que se le otorga al diseño de propuestas pedagógicas específicas.

-El FinEs se consolidó como la principal línea de política educativa de diversos planes y programas sociales bajo el enfoque de políticas sociales integrales, y de esta forma expresó su potencial de articulador de diversos actores territoriales y gubernamentales. Este potencial se desplegó con intensidad en la PBA y con debilidad en la CABA.

- La característica compartida de tensiones y desafíos en relación a la estabilidad docente dentro de los programas a término se manifiestan diferencialmente según las jurisdicciones. En el caso del Plan FinEs se evidencia una mayor fragilidad en la CABA en donde no se registra homologación de las condiciones salariales como sí es factible encontrarla en la PBA.

-Finalmente, las posibilidades de diseñar y promover políticas educativas para la modalidad de jóvenes y adultos encuentran aspectos dinamizadores en las lógicas de inscripción territorial y en los formatos no escolarizantes. 
En relación al estudio del FinEs nos resulta relevante visibilizar las capacidades de dotar de sentidos propios que tienen las jurisdicciones en el marco de un sistema de organización federal, atravesado por distintos signos políticos en los ámbitos provinciales o jurisdiccionales. Así, las disputas, los sentidos, el poder y las coaliciones a las que refieren Oszlak y O’Donnell (1981) se manifiestan no solamente al interior del Estado Nacional, sino también entre los distintos niveles de gobierno que conducen los distintos estados subnacionales.

Entonces, debemos entender que el FinEs se configura de la manera descripta en tanto política de alcance nacional, articulación federal, e implementación local. Nacional en tanto el programa es propuesto y financiado en gran parte por el Estado nacional, quien a través del mismo manifiesta su preocupación por los altos índices de no terminalidad educativa y su intencionalidad de asumir la responsabilidad de la obligatoriedad del nivel secundario establecida por la LEN. De articulación federal porque es a través de la suscripción de convenios específicos entre las provincias y la Nación que se financia y ejecuta el programa. $Y$ finalmente de implementación local, ya que será responsabilidad de cada jurisdicción la definición de las características concretas que asuma la apertura de sedes, la designación de docentes, y las condiciones de trabajo asociadas. Es en este proceso local donde se observa cómo las políticas son reapropiadas y resignificadas, y donde, en última instancia, estas políticas en tanto expresión del Estado cobran realidad y materialidad para los ciudadanos de a pie.

\section{Notas}

(1) Los Centros de Orientación y Apoyo (COA) existen en el ámbito de la pprovincia de Buenos Aires desde 2003.

(2) "Programa de Respaldo a Estudiantes Argentinos" (PROG.R.ES.AR), destinado a jóvenes de entre 18 y 24 años que no hayan comenzado o culminado sus estudios y no se encuentren trabajando. El Plan FinEs se contempla como una de las posibles ofertas educativas para acreditar la realización de estudios que el PROG.R.ES.AR requiere en calidad de contraprestación por la asignación del subsidio asociado.

(3) En 2013 se incluye como línea programática dentro del Programa "Ingreso Social con Trabajo-Argentina Trabaja", destinado especialmente a mujeres sin trabajo registrado, que perciben la Asignación Universal por Hijo (AUH).

(4) En el ámbito de la provincia de Buenos Aires se superponen los servicios educativos destinados a jóvenes y adultos dependientes de la Dirección Provincial de Nivel Secundario (Bachilleratos de Adultos) y aquellos dependientes de la Dirección Provincial de Adultos (Centros Educativos de Nivel Secundarios-CENS).

(5) De estos 230.000, alrededor de 170.000 mil se inscribían en secundarias de adultos; 30.000 en CENS y los restantes se encontraban distribuidos entre las otras ofertas educativas como el Bachillerato Semipresencial y los Centros de Escolarización Secundaria para Adolescentes y. Jóvenes (CESAJ). 
(6) Los datos sobre cantidad de estudiantes en CENS, bachilleratos de adultos y plan Fines corresponde a la matríc ula de 2014 y fueron obtenidos a través del equipo de la Dirección de Educación de Adultos de la PBA.

(7) Cabe aclarar que el Programa PRIST-AT se implementó en su primera etapa sólo en 32 distritos de la PBA. Sí se incluirá a CABA en la ejecución del Programa "Ellas Hacen", que no tendrá tampoco el impacto y las dimensiones esperadas inicialmente según las entrevistas realizadas a funcionarios responsables de la implementación del PEH en CABA.

(8) Mientras en la provincia de Buenos Aires encontramos un mis mo signo político (Frente para la Victoria) gobernando en el orden nacional y subnacional, para el caso de la Ciudad Autónoma de Buenos Aires encontramos un partido político diferente gobernando en el orden local (Partido Propuesta Republicana-PRO).

(9) Cerca de 500.000 personas según los datos del CENSO 2010.

(10) Para más información: Kirchesky, 2012.

(11) Años más tarde, en 2013, se generará un articulación similar con el Programa "Ellas Hacen" (PEH) que incluirá al Plan FinEs como una modalidad (obligatoria) de contraprestación de las transferencias monetarias percibidas y por eso, en la mayoría de los casos, se constituirán lo que se han llamado "comisiones puras", es decir comisiones conformadas solo por las titulares del PEH.

(12) Los criterios que sustentan el ejercicio comparativo entre ambas jurisdicciones han sido explicitados en la presentación de este trabajo.

(13) Los contenidos a contemplar en dichos proyectos se encuentran delimitados por la Resolución DGCyE 6321/95, para el caso de la PBA, y por la Resolución 206/83, para el de la Ciudad. En ambos casos los planes de estudios que se utilizan para el FinEs son los mismos que los que se establecen para las propuestas de educación secundaria de la modalidad de jóvenes y adultos de cada jurisdicción.

\section{Normativa analizada}

Disposición № 99. Correspondencia entre diferentes Planes de Estudios y el Plan Provincial de Finalización de Estudios Obligatorios, Reservorio institucional del ABC, Secretaría de Educación de la Dirección General de Cultura y Educación, La Plata, Buenos Aires, Argentina, 8 de agosto del 2012.

Ley № 24195. Ley Federal de Educación. Información Legislativa (InfoLEG). Buenos Aires, Argentina, 14 de abril de 1993.

Ley № 26206. Ley de Educación Nacional. Información Legislativa (InfoLEG). Buenos Aires, Argentina, 14 de diciembre de 2006.

Resolución № 66. Plan de Finalización de Estudios Primarios y Secundarios-“FinEs”. Consejo Federal de Educación. Buenos Aires, Argentina, 28 de octubre de 2008.

Resolución № 206. Planes de Estudio de los Centros Educativos del Nivel Secundario. Normativa sobre Planes de Estudio de Nivel Medio, Ministerio de Educación del G.C.A.B.A. Capital Federal, Argentina, 2 de marzo de 1983. 
Resolución № 444. Plan Provincial de Finalización de Estudios Obligatorios, Información Legislativa Provincial (InfoLEG), Dirección General de Cultura y Educación, La Plata, Buenos Aires, Argentina, 20 de octubre del 2010.

Resolución № 737. Bachillerato para Adultos a Distancia, Dirección General de Cultura y Educación, La Plata, Buenos Aires, Argentina, marzo de 2007.

Resolución № 3520. Plan FinEs Secundaria en la Provincia de Buenos Aires, Información Legislativa Provincial (InfoLEG), Dirección General de Cultura y Educación, La Plata, Buenos Aires, Argentina, 16 de abril del 2012.

Resolución № 3789. SE MODIFICA LA RESOLUCIÓN 8644-MEGC-09-PLAN FINES, Boletín Oficial de la Ciudad de Buenos Aires, Ministerio de Educación del Gobierno de la Ciudad, Ciudad Autónoma de Buenos Aires, 29 de junio de 2009.

Resolución № 6321. Plan de Estudios del Bachillerato de Adultos. Información Legislativa Provincial (InfoLEG). Dirección General de Cultura y Educación La Plata, Buenos Aires, Argentina, 7 de diciembre de 1995.

Resolución № 8644. Implementación en el ámbito de la Ciudad el Plan de Finalización de Estudios Primarios y Secundarios-PLAN FINES, Boletín Oficial de la Ciudad de Buenos Aires, Ministerio de Educación del Gobierno de la Ciudad, Ciudad Autónoma de Buenos Aires, 31 de diciembre de 2008.

\section{Bibliografía}

Aguilar Villanueva, L. (2007). El aporte de la Política Pública y de la Nueva Gestión Pública a la Gobernanza. Revista del CLAD Reforma y Democracia, 39. Recuperado en http://old.clad.org/portal/publicaciones-del-clad/revista-clad-reformademocracia/articulos/039-octubre-2007/0057201

Cabrera, M. E. (2006). El campo de la educación de adultos. Su diversidad conceptual y política. En Brusilovsky, S. (Ed.). Educación Escolar de Adultos (pp. 9-15). Buenos Aires, Argentina: Ed. Noveduc.

Feldfeber, M. (noviembre-diciembre de 1997). La propuesta educativa neoliberal. Revista Espacios de crítica y producción, 22. Facultad de Filosofía y Letras, Universidad de Buenos Aires.

Freire, P. (2002). Pedagogía del Oprimido. Buenos Aires, Argentina: Siglo Veintiuno editores. 
Gentili, P. (1998). El consenso de Washington y la crisis de la educación en América Latina. En Álvarez Uria, F.y otros (Comp). Neoliberalismo versus democracia (pp. 103-129). Madrid, España: La Piqueta.

Instituto Nacional de Estadística y Censos (INDEC) (2012). Censo nacional de población, hogares y viviendas 2010: censo del Bicentenario: resultados definitivos. Serie B, 2. Buenos Aires, Argentina.

Kirchner, A. (2007). La Bisagra. Políticas Sociales en Acción. Buenos Aires, Argentina: Ministerio de Desarrollo Social de la Nación.

Kirchesky, M. (coord.) (2012). Educación Secundaria para Jóvenes y Adultos. Aportes acerca de la oferta, sus alcances y principales indicadores. Recuperado de https://goo.gl/S4BHrB

Misirilis, G. (2009). Deudas y desafíos en la educación de jóvenes y adultos: una mirada desde un enfoque político-pedagógico. Documento IDIE del MERCOSUR, Paraguay. Recuperado de http://www.oei.es/historico/noticias/spip.php?article4548

Oszlak, O. y O’Donnell, G. (1981). Estado y Políticas Estatales en América Latina: hacia una estrategia de investigación. Buenos Aires, Argentina: Centro de Estudios de Estado y Sociedad (CEDES), CLACSO.

Rodríguez, L. (Comp.) (2013). Educación popular en la historia reciente en Argentina y América Latina. Aportes para balance y prospectiva. Buenos Aires, Argentina: APPEAL.

Tamayo Sáez, M. (1997). El análisis de las políticas públicas. En Bañon, R. y Carrillo, E. (Comps.). La nueva Administración pública (pp. 281-312). Madrid, España: Alianza Universidad. 